\title{
PS4:71 IGA ANTI-PHOSPHOLIPID ANTIBODIES IN SWEDISH CASES WITH SYSTEMIC LUPUS ERYTHEMATOSUS: ASSOCIATIONS WITH DISEASE PHENOTYPES, VASCULAR EVENTS AND DAMAGE ACCRUAL
}

${ }^{1} \mathrm{M}$ Frodlund, ${ }^{2} \mathrm{~A}$ Vikerfors, ${ }^{2} \mathrm{G}$ Grosso, ${ }^{1} \mathrm{~T}$ Skogh, ${ }^{1} \mathrm{~J}$ Wetterö, ${ }^{3} \mathrm{~K}$ Elvin, ${ }^{2} \mathrm{G}$ Gunnarsson, ${ }^{1} \mathrm{~A}$ Kastbom, ${ }^{4} \mathrm{O}$ Dahlström, ${ }^{5} \mathrm{~J}$ Rönnelid, ${ }^{2} \mathrm{E}$ Svenungsson, ${ }^{1} \mathrm{C}$ Sjöwall. ${ }^{1}$ Divisoin of Neuro and Inflammation Sciences, Department of Clinical and Experimental Medicine, Linköping University, Linköping, Sweden; ${ }^{2}$ Unit of Rheumatology, Department of Medicine Solna, Karolinska University Hospital, Stockholm, Sweden; ' ${ }^{3}$ Unit of Clinical Immunology, Department of Clinical Immunology and Transfusion Medicine, Karolinska Institutet, Stockholm, Sweden; ${ }^{4}$ Swedish Institute for Disability Research, Department of Behavioural Sciences and Learning, Linköping University, Linköping, Sweden; ${ }^{5}$ Department of Immunology, Genetics and Pathology, Uppsala University, Uppsala, Sweden

\subsection{6/lupus-2018-abstract.117}

Objectives IgG- and IgM-class anti-cardiolipin antibodies (aCL) and lupus anticoagulant (LA) are included in the 1997 update of the American College of Rheumatology (ACR-97) systemic lupus erythematosus (SLE) classification criteria. Despite limited evidence, IgA-aCL and IgA anti- $\beta 2$-glycoprotein-I (anti- $\beta$ 2GPI) were included among the 2012 Systemic Lupus International Collaborating Clinics classification criteria. The present study was undertaken to evaluate $\operatorname{IgG}-/ \operatorname{IgA}-/ \operatorname{IgM}-\mathrm{aCL}$ and anti$\beta 2$ GPI occurrence in relation to disease phenotype, smoking habits, pharmacotherapy, APS-related events, and organ damage among Swedish SLE patients.

Methods 526 SLE patients meeting ACR-97 were included. Blood donors and patients with rheumatoid arthritis or primary Sjögren's syndrome served as controls. Serum anti-phospholipid antibodies (aPL) were analysed by enzymeimmunoassays.

Results 76 (14\%) SLE cases fulfilled the Sydney APS-criteria, and at least $1 \mathrm{aCL} /$ anti- $\beta 2 \mathrm{GPI}$ isotype (IgG/IgA/IgM) occurred in 138 SLE patients (26\%). 44 (8\%) of the SLE cases had

A
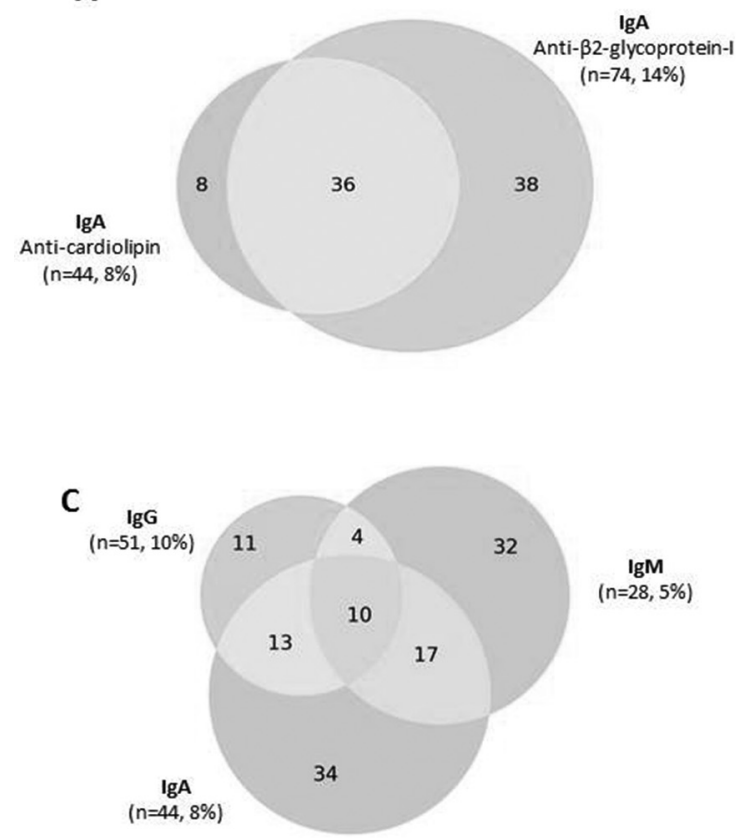

IgA-aCL, of whom 20 (4\%) lacked IgG-/IgM-aCL. 74 (14\%) tested positive for IgA anti- $\beta 2 \mathrm{GPI}, 34(6 \%)$ being seronegative regarding $\mathrm{IgG} / \mathrm{IgM}$ anti- $\beta 2 \mathrm{GPI} .6(1 \%)$ had manifestations compatible with APS and were seropositive regarding IgA-aCL and/or IgA anti- $\beta 2$ GPI in absence of $\operatorname{IgG/IgM-aPL}$ and LA. Positive LA- and IgG-aPL tests associated with most APSrelated events and organ damage. Exclusive IgA anti- $\beta 2$ GPI occurrence associated inversely with Caucasian ethnicity and photosensitivity. Nephritis, smoking, LA-positivity and statin/ corticosteroid-medication associated strongly with organ damage, whereas ongoing hydroxychloroquinemedication was protective.

Conclusions IgA-aPL is not uncommon in SLE (16\%). Exclusive IgA anti- $\beta 2 \mathrm{GPI} \pm \mathrm{IgA}$ aCL associated with non-Caucasian ethnicity. IgA-aPL analysis may be of additional value among clinically suspected APS-patients testing negative for other isotypes of aPL and LA.

\section{PS4:72 ANTIPHOSPHOLIPID ANTIBODIES AND AUTOIMMUNE HEMOLITIC ANAEMIA IN SYSTEMIC LUPUS ERYTHEMATOSUS: A CASE-CONTROL STUDY}

${ }^{1} \mathrm{~F}$ Crisafulli, ${ }^{1} \mathrm{~L}$ Andreoli, ${ }^{2} \mathrm{~F}$ Franceschini, ${ }^{2} \mathrm{M}$ Frassi, ${ }^{2} \mathrm{M}$ Fredi, ${ }^{2} \mathrm{C}$ Nalli, ${ }^{1} \mathrm{~A}$ Tincani. ${ }^{1}$ Rheumatology and Clinical Immunology Unit, Spedali Civili and University of Brescia, Italy; ${ }^{2}$ Rheumatology and Clinical Immunology Unit, Spedali Civili of Brescia, Italy

\subsection{6/lupus-2018-abstract.118}

Purpose Hemolitic anaemia with reticulocitosis is included in ACR and SLICC classification criteria of Systemic Lupus Erithematosus (SLE). Some studies have assessed the relationship between antiphospholipid antibodies (aPL) and autoimmune hemolitic anaemia (AIHA) in SLE patients. Some of them had describe a correlation with the presence of Lupus
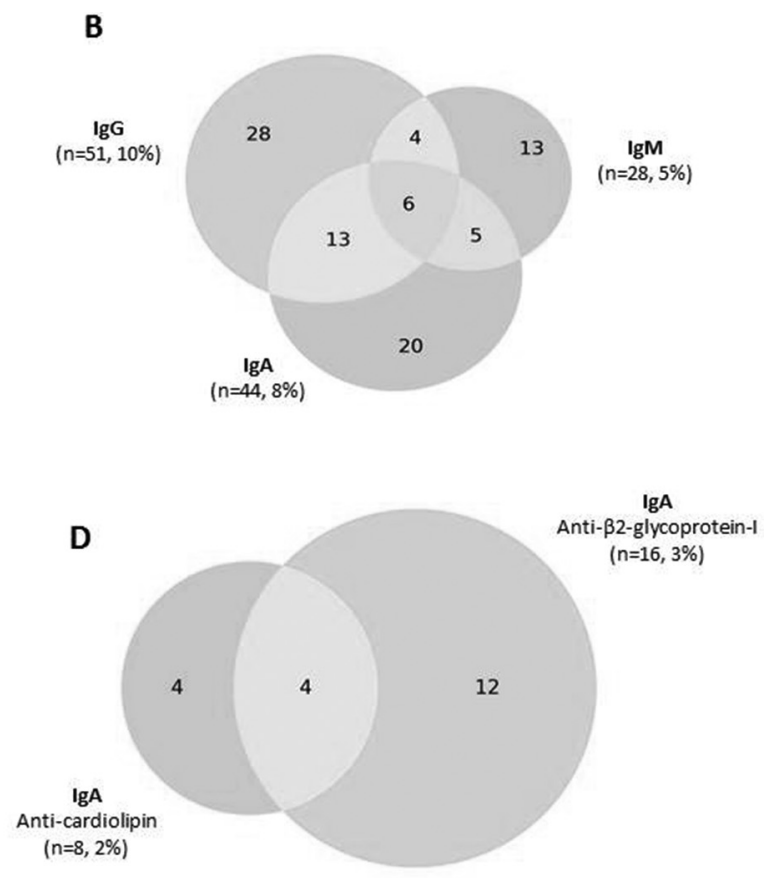

Abstract PS4:71 Figure 1 A: distribution of IgA aCL and IgA anti- $\beta_{2}$ GPI positive cases in the full SLE cohort. 82 (16\%) of the SLE cases had IgA positivity, $44(8 \%)$ of aCL and $74(14 \%)$ of anti- $\beta_{2} \mathrm{GPI}$ type. B: Distribution of lgG/A/M isotypes of aCL in the SLE cohort. 89 (17\%) SLE cases were positive for at least one $\mathrm{aCL}$ isotype. C: Distribution of $\mathrm{IgG} / \mathrm{A} / \mathrm{M}$ isotypes of anti- $\beta_{2} \mathrm{GPI}$ in the SLE cohort. 121 (23\%) SLE cases were positive for at least one anti- $\beta_{2} \mathrm{GPI}$ isotype. D: Distribution of exclusively $\operatorname{lgA}$ aCL and IgA anti- $\beta_{2} \mathrm{GPI}$ positive cases in the SLE cohort. 20 (4\%) of the SLE cases had IgA positivity, $8(2 \%)$ of $\mathrm{aCL}$ and $16(3 \%)$ of anti- $\beta_{2} \mathrm{GPI}$ type 
Abstract PS4:72 Table 1

\begin{tabular}{|l|c|c|r|}
\hline & $\begin{array}{c}\text { AlHA (\%) } \\
\mathbf{n}=16\end{array}$ & $\begin{array}{c}\text { CONTROLS (\%) } \\
\mathbf{n = 3 2}\end{array}$ & p value \\
\hline aPL positivity & $13(81)$ & $14(44)$ & $\mathbf{0 , 0 1 6 4}$ \\
\hline Single aPL positivity & $2(13)^{*}$ & $4(13)$ & 1,0000 \\
\hline Double aPL positivity & $8(53)^{*}$ & $7(22)$ & $\mathbf{0}, 0459$ \\
\hline Triple aPL positivity & $1(7)^{*}$ & $3(9)$ & 1,0000 \\
\hline aCL & $6(39)$ & $8(25)$ & 0,5026 \\
\hline aCL IgG & $4(25)$ & $8(25)$ & 1,0000 \\
\hline low-titer & $2(13)$ & $2(6)$ & 0,5921 \\
\hline medium-titer & $2(13)$ & $5(16)$ & 1,0000 \\
\hline high-titer & $0(0)$ & $1(3)$ & 1,0000 \\
\hline aCL IgM & $4(25)$ & $4(13)$ & 0,4130 \\
\hline low-titer & $3(19)$ & $3(9)$ & 0,3858 \\
\hline medium-titer & $1(6)$ & $1(3)$ & 1,0000 \\
\hline high-titer & $0(0)$ & $0(0)$ & 1,0000 \\
\hline $\boldsymbol{\beta}_{2}$ GPI & $10(63)$ & $13(41)$ & 0,2221 \\
\hline $\boldsymbol{\beta}_{2}$ GPI IgG & $8(50)$ & $10(31)$ & 0,2258 \\
\hline low-titer & $6(39)$ & $6(19)$ & 0,1776 \\
\hline medium-titer & $2(13)$ & $1(3)$ & 0,2544 \\
\hline high-titer & $0(0)$ & $3(9)$ & 0,5412 \\
\hline $\boldsymbol{\beta}_{2}$ GPI IgM & $6(39)$ & $6(19)$ & 0,1776 \\
\hline low-titer & $4(25)$ & $5(16)$ & 0,4566 \\
\hline medium-titer & $2(13)$ & $0(0)$ & 0,1064 \\
\hline high-titer & $0(0)$ & $1(3)$ & 1,0000 \\
\hline IgG (aCL+ $\boldsymbol{\beta}_{2}$ GPI) & $12(75)$ & $18(56)$ & 0,3431 \\
\hline IgM (aCL+ $\boldsymbol{\beta}_{2}$ GPI) & $10(63)$ & $10(31)$ & 0,5908 \\
\hline LAC & $8(50)^{*}$ & $5(16)$ & 0,0131 \\
\hline & & &
\end{tabular}

${ }^{*} \mathrm{n}=15$

anticoagulant (LAC) as other highlighted the association with a positive anti-B2glycoprotein I (B2GPI) IgG or anti-cardiolipin (aCL) IgM. The purpose of our preliminary study is to analyse and compare aPL positivity in two groups of SLE patients, one with a history of AIHA and one without it.

Methods The retrospective analysis of our cohort of 514 SLE patients showed that 16 subjects fulfil a completely documented diagnosis of AIHA at some stage of their disease. These subjects and 32 consecutive SLE patients without any haematological manifestations were included in this study. Clinical and serological data were obtained from medical records of our clinic. We compared the prevalence of aPL, their type (aCL IgG and IgM, B2GPI IgG and IgM, LAC) and titre using Fisher exact test in the two groups of patients.

Results Lupus patients with AIHA had a significantly higher frequency of aPL positivity compared with SLE patients without AIHA ( $81 \%$ vs 44\%, p value $=0,0164)$. Furthermore, double aPL positivity was detected in $53 \%$ of patients with AIHA as in the control group was $22 \%$ ( $p$ value $=0,0459$ ). The positivity of aCL and B2GPI was higher in the group of patients with history of AIHA than in the control group (39\% vs $25 \%$ and $63 \%$ vs $41 \%$, respectively) but the difference was not statistically significant; table1 details the results of each antibody isotype and titres. The presence of LAC was significantly associated with history of AIHA ( $p$ value $=0,0131$ ).
Conclusions In this control-case study the frequency of aPL was higher in SLE patients with a history of AIHA. In particular, in this group we found a higher frequency of double aPL positivity. Between aPL, LAC had the strongest association with AIHA. The absence of statistical difference considering aCL and B2GPI positivity and titres could be due to the low sample size.

\section{PS4:73 EFFECT OF HYDROXYCHLOROQUINE AND PREDNISONE TREATMENT ON ANTIPHOSPHOLIPID TITERS IN SLE}

${ }^{1} \mathrm{M}$ Avci, ${ }^{2} \mathrm{~L}$ Magder, ${ }^{3} \mathrm{M}$ Petri. ${ }^{1}$ Istanbul Faculty of medicine, Istanbul, Turkey; ${ }^{2}$ University of Maryland School of Medicine, Baltimore, MD, USA; ${ }^{3}$ Johns Hopkins University School of Medicine, Baltimore, MD, USA

\subsection{6/lupus-2018-abstract.119}

Background Antiphospholipid antibodies in SLE may be affected by disease activity and treatment, but past studies have been conflicting. We investigated the association of titers of antiphospholipid antibodies with disease activity and therapy.

Methods 943 SLE patients, who had at least 10 quarterly visits for testing for each anticardiolipin isotype (IgG, $\operatorname{IgM}$ and IgA) and dRVVT, were included in the study. Disease activity 